Stephen R. H. Langton, Alex H. McIntyre, Peter J. B. Hancock \& Helmut Leder (2018) Saccades and smooth pursuit eye movements trigger equivalent gaze-cued orienting effects, The Quarterly Journal of Experimental Psychology, 71 (9), pp. 1860-1872. Copyright (C) Experimental Psychology Society 2017. Reprinted by permission of SAGE Publications.

\title{
Saccades and smooth pursuit eye movements trigger equivalent gaze-cued
}

\section{orienting effects}

\author{
Stephen R. H. Langton, Alex H. McIntyre ${ }^{1}$, Peter J. B. Hancock \\ Faculty of Natural Sciences, University of Stirling, UK \\ and
}

Helmut Leder

Department of Basic Psychological Research and Research Methods, University of Vienna

Running Head: Eye movement type and gaze-cueing

Word count: 7996

\section{Corresponding author:}

Dr Stephen R. H. Langton

Faculty of Natural Sciences

University of Stirling

Stirling

FK9 4LA

UK

Email: stephen.langton@stir.ac.uk

Tel: +44 (0)1786 467659

\section{Acknowledgements}

This research was supported by the Economic and Social Research Council [grant number

ES/1034803/1]. We thank Matthew Paul for his assistance in generating the stimuli used in the experiments.

\section{Footnote}

1. Alex McIntyre is now at the School of Life, Sport and Social Sciences, Sighthill Court, Edinburgh Napier University, Edinburgh, UK. 


\begin{abstract}
Research has established that a perceived eye gaze produces a concomitant shift in a viewer's spatial attention in the direction of that gaze. The two experiments reported here investigate the extent to which the nature of the eye movement made by the gazer contributes to this orienting effect. On each trial in these experiments participants were asked to make a speeded response to a target that could appear in a location toward which a centrally presented face had just gazed (a cued target), or in a location that was not the recipient of a gaze (an uncued target). The gaze cues consisted of either fast saccadic eye movements or slower smooth pursuit movements. Cued targets were responded to faster than uncued targets, and this gaze-cued orienting effect was found to be equivalent for each type of gaze shift both when the gazes were un-predictive of target location (Experiment 1) and counterpredictive of target location (Experiment 2). The results offer no support for the hypothesis that motion speed modulates gaze-cued orienting. However, they do suggest that motion of the eyes per se, regardless of its type, may be sufficient to trigger an orienting effect.
\end{abstract}

Keywords: gaze-cued attention; gaze cueing; gaze following; gaze-cued orienting; eye gaze; eye movement type 
A wealth of research over the last two decades has established the existence of a mechanism that serves to deploy covert attentional resources automatically in the direction in which we see others look - so-called gaze-cued orienting of attention (Driver et al., 1999; Friesen \& Kingstone, 1998; Langton \& Bruce, 1999; for a review see Frischen, Bayliss, \& Tipper, 2007). Such a mechanism may have evolved to exploit the fact that people tend to look at significant objects or events in their environments, such as potential threats or mating opportunities, the referents of their remarks, the targets of their planned actions, their conversational partners, and so on. Most of the work in this area has used either static gazes or simulations of saccadic gazes as the critical directional cues. The present work uses video recordings of real dynamic gazes and examines whether the type of gaze - the nature of the motion that brings the eyes to bear on an object of interest - influences the mechanism that sub-serves gaze-cued orienting of attention.

The experiments that have demonstrated that gazes trigger shifts of attention have used a variant of Posner's pre-cueing paradigm (Posner, 1980). On each of a number of trials in these experiments a face is presented in the centre of a screen looking to the left or right. Shortly after this, a target letter is presented to the left or right of the face. Participants are asked to make a speeded keypress response contingent upon either the detection of the letter, its identity, or its location. The typical finding is that reaction times (RTs) are faster for targets that appeared in the looked-at (cued or valid) location than for targets that appeared in the opposite (uncued or invalid) location (Driver et al., 1999; Friesen \& Kingstone, 1998; Langton \& Bruce, 1999). It is generally agreed that this gaze cueing effect occurs because the observed gaze direction triggers a shift of attention along the line of regard of the gazing face. Furthermore, gaze-cueing is considered by some to be a kind of reflex because it occurs when participants know that the likelihood of a target appearing in a gazed-at location is $50 \%$, or even when it is actually more likely that a target will appear in an uncued location 
(Driver et al., 1999; Friesen, Ristic, \& Kingstone, 2004; Tipples, 2008). Similarly, the findings that gaze-cueing occurs when participants are explicitly asked to ignore the gaze cues (Langton \& Bruce, 1999), or when they are concurrently performing fairly demanding secondary tasks (Hayward \& Ristic, 2013; Law, Langton, \& Logie, 2010; but see Bobak \& Langton, 2015) are also taken as evidence for the automaticity of the effect.

The movement of the eyes is a factor in gaze-cued orienting that has attracted relatively little research. Many of the relevant studies have used either schematic faces or photorealistic faces displaying static gazes so as to avoid any confounding contribution of motion signals to the orienting effect (Driver et al., 1999; Friesen \& Kingstone, 1998; Langton \& Bruce, 1999; Law et al., 2010; Ristic \& Kingstone, 2005; Schuller \& Rossion, 2004). Others have included such signals in the form of actual motion of the eyes of a copresent gazer (Lachat, Conty, Hugueville, \& George, 2012), or apparent motion of the eyes induced by first displaying a static image of a face with a direct gaze and then replacing it with an otherwise identical image save for the lateral displacement of the iris and pupil region to the left or right (Bayliss \& Tipper, 2006; Bayliss, Griffiths, \& Tipper, 2009; Bobak \& Langton, 2015; Quadflieg, Mason, \& Macrae, 2004; Schuller \& Rossion, 2001). Gazecued orienting is robust in all these cases, so it is clear that motion is not necessary to trigger the effect.

It is perhaps natural to conclude from this evidence that gaze cueing is driven by some static cue, or set of cues, in the eye, which correlate with gaze direction. Indeed, much of the work on gaze perception has emphasized static features. For example, early accounts implied that the task involved segmenting the iris from the sclera and measuring its location relative to some fixed feature on the face such as the corner of the eye (Anstis, Mayhew, \& Morley, 1969; Cline, 1967; Gibson \& Pick, 1963). More recent work has suggested that, in addition, gaze direction can be computed through the degree of luminance contrast over the 
eye region (Ando, 2004; Langton, Watt, \& Bruce, 2000; Watt, Craven, \& Quinn, 2007). Finally, Calder and colleagues have shown that different populations of STS cells are tuned to different gaze directions, as signaled by particular static configurations of eye features (Calder, Beaver, Winston, \& Dolan, 2007; Jenkins, Beaver, \& Calder, 2006).

While the static arrangement of eye features may well be critical for extracting precise information about gaze angle, it is not hard to imagine that a change in this arrangement (i.e., motion), or the rate of change of the configuration (i.e., motion speed) might also serve as cues for attention shifting in response to gaze. For example, the change, or rate of change, of either the luminance contrast in the eye region or of the spatial position of the iris might serve as rapidly available low-level cues to the side of space in which the gazer's attention is being directed without carrying any information about the precise line of regard of the gaze. Indeed, the evidence suggests that gaze-cued orienting is quite imprecise and may, in fact, generalize to the entire cued hemifield rather than a specific cued location (Wiese, Zwickel, \& Müller, 2013).

In summary, although it is clear that static gazes are capable of initiating gaze-cued attention, it is nevertheless possible that the motion of the eyes and the speed of that motion contribute to the gaze-cued orienting effect. The experiments reported here investigate the latter of these possibilities using naturally occurring gaze cues.

There are, in fact, only two types of naturally occurring, voluntarily executed eye movements that are visible to observers: saccades and smooth pursuit movements. Saccades are used to fixate stationary objects in the environment; they are fast, with their speed being essentially invariant for a given amplitude of gaze shift (around $500^{\circ} / \mathrm{sec}$ for a shift of $30^{\circ}$ ). Smooth pursuit movements are used to track moving objects and they are much slower than saccades (up to around $30^{\circ} / \mathrm{sec}$ ). To the best of our knowledge, the only types of eye movements to have been studied in the context of gaze-cued orienting are either actual 
saccades (Lachat et al., 2012) or simulations of saccades (Bayliss et al., 2009; Bayliss \& Tipper, 2006; Bobak \& Langton, 2015; Quadflieg et al., 2004; Schuller \& Rossion, 2001). In Experiments 1 and 2 we test whether the gaze-cued orienting effect extends to smooth pursuit gazes and to actual video clips of real saccadic eye movements.

From a physical perspective, a saccadic gaze clearly involves a greater rate of change in the features of the eye that are thought to be used to perceive gaze direction (e.g., spatial position of the iris/pupil region, or the relative luminance of the scleral regions on either side of the iris) than does a smooth pursuit movement. If the system that triggers gaze-cued orienting takes eye motion as a cue, then we would predict that saccades will be more potent stimuli than pursuit movements - potent in the sense of being more likely to engage a viewer's attention system, or in causing the deployment of more of a viewer's available attentional resource. Each would result in an enhanced gaze-cueing effect for saccades relative to smooth pursuit eye movements.

The same prediction might also be derived from functional considerations. A significant event in the environment such as the appearance of a new object would likely attract a saccadic eye movement from someone for whom that event is visible. If that object started to move, or was already moving when targeted by a saccadic eye movement, a viewer might then track the object with a smooth pursuit movement. From the perspective of someone observing the gazer, an observed saccadic movement will therefore often indicate something important in the environment that might be worthy of his or her own scrutiny; a pursuit movement, on the other hand, will generally appear after a saccade has been made to fixate the moving object, and so may be less informative to an observer. Again, the prediction is that saccadic shifts may result in an enhanced gaze cueing effect compared to pursuit gazes, even though both shifts may result in the fixation of the same location in the environment. 
Demonstrating this kind of sensitivity to the type of eye movement would help address one of the puzzles associated with the idea that viewing another's gaze triggers an automatic shift of attention, which is that it seems counter to everyday observation: listeners in dyadic interactions spend much of their time looking at speakers' faces (Argyle, 1988; Kendon, 1967), and seldom initiate overt attention shifts in response to witnessing relatively frequent speaker gaze aversions (Langton, McIntyre, \& Hancock, 2017). Of course, it may be that while such gazes fail to initiate overt gaze-following responses, they nevertheless continue to trigger automatic covert attention shifts, but this would seem very inefficient. Another possibility is that gaze-cued orienting of attention is still automatic and stimulusdriven, but it is sensitive to certain physical features of gazes that may correlate with those gazes that are, or are not, worth following. The experiments reported here explore whether the motion speed associated with an eye movement - or more precisely, the natural kind distinction between saccades and smooth pursuit movements - represents one such feature.

\section{Experiment 1}

In Experiment 1 participants were asked to make a speeded discrimination response to one of two target stimuli that could appear in either a gaze-cued or an uncued location. The cues in this experiment were short video clips of a male individual who was filmed making a saccadic gaze shift and a smooth pursuit movement. In order to investigate the time course of the gaze-cued orienting effect, the time between the onset of each of the gaze cues and the onset of the target stimuli - the stimulus onset asynchrony (SOA) - was also manipulated. Previous studies have suggested that gaze-cued orienting can emerge as soon as $100 \mathrm{~ms}$ following cue onset (Friesen \& Kingstone, 1998; Hill, Patel, Gu, Seyedali, \& Bachevalier, 2010), while others have suggested that the effect is not observable until around $300 \mathrm{~ms}$ after cue onset (e.g., Driver et al., 1998). For these reasons, SOAs of $125 \mathrm{~ms}, 333 \mathrm{~ms}$ and $667 \mathrm{~ms}$ were used in this study. 
Based on the arguments presented above, we predicted that saccadic gazes would be more potent cues for the attention system than pursuit gazes. This would manifest itself as a larger difference between RTs on uncued and cued trials for saccadic gazes than for pursuit gazes, either because the former, on average, result in the deployment of more attention resource in the direction of gaze, and/or attention shifts occur on proportionately more trials involving saccades than pursuit gazes. Furthermore, we expected this difference to manifest itself more at shorter SOAs, where gaze movements are either still ongoing or just completed, than at the longest SOA $(667 \mathrm{~ms})$ where the final configuration of the eyes may become more important.

\section{Method}

\section{Participants}

These were 26 undergraduate students at the University of Stirling (9 males). The average age of the sample was 23 , with a range from 19 to 44 . All had normal or correctedto-normal vision, and written informed consent was obtained from all participants.

\section{Materials and apparatus}

Video clips were prepared depicting a white male individual performing saccadic and smooth pursuit gaze shifts. This individual was one of the authors (SL), a member of staff in the Department of Psychology who would likely have been familiar to some of participants. For the recording of each type of gaze the actor sat in front of a screen with his chin in a chin rest to reduce head movements that tend to occur with gaze movements. Recordings were made using a digital camcorder recording at 24 frames per second, which was situated between the actor and the screen just below the actor's eye level. For the pursuit movements the actor tracked a large dot that was projected onto the screen. This dot was initially presented in the center of the screen where it was fixated by the actor. The dot then moved at a constant rate along a horizontal line from the center to the left hand side of the screen, and 
then returned to the right hand side of the screen along the same trajectory. The dot continued to move backwards and forwards along this horizontal line while the actor tracked the movement. The distance from the center to either side of the screen subtended approximately 30 degrees of the gazer's horizontal visual angle. For the saccadic eye movements, the actor first fixated the center of the screen and then made saccades to fixate targets located at the left and right hand sides of the screen at the same locations as the endpoints of the trajectories of the moving targets used to record the pursuit gazes.

The recordings were then edited using Adobe Premier to produce full-colour clips; two clips contained saccades and two contained pursuit movements (leftwards and rightwards movements for each type of gaze). Each clip began with the actor staring straight ahead for $1000 \mathrm{~ms}$ (24 frames) followed by either a saccade or a pursuit to the left or right. The duration of the saccadic and pursuit eye movements were $125 \mathrm{~ms}$ (i.e., 3 frames at 24 frames per second) and $667 \mathrm{~ms}$ (16 frames) respectively (see Figure 1). The total duration of each of the saccade and pursuit clips was therefore $1125 \mathrm{~ms}$ and $1667 \mathrm{~ms}$ respectively. When presented in the main part of the experiment, the faces in each of the clips measured $4.1^{\circ}$ of horizontal visual angle, by $6^{\circ}$ vertically, and were viewed from a distance of $47.5 \mathrm{~cm}$ by participants using a chin rest.

The targets in this experiment were the letters T and F. These were displayed in 18 point Arial font in white on a black background. Target letters could appear $6^{\circ}$ to the left or right of fixation. Responses were recorded using the " 8 " and " 2 " keys on the keypad area of a standard keyboard. 

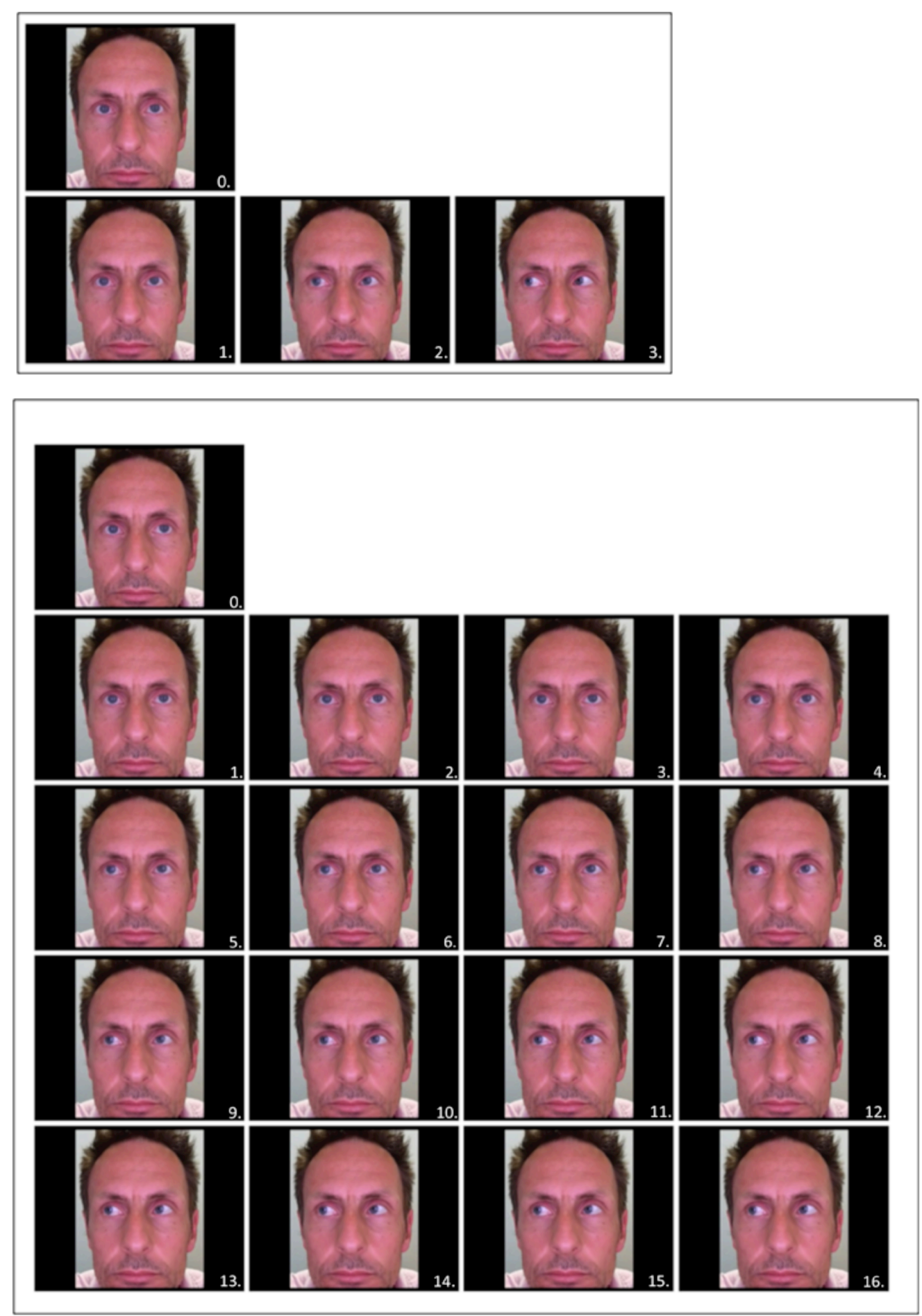

Figure 1. Illustrations of the sequence of frames used for the saccade (top panel) and pursuit (bottom panel) eye movements. Each clip began with 24 repetitions of the frame depicting a straight ahead gaze (labeled frame 0 ), followed by three frames for the saccade (labeled 1-3) and 16 frames for the pursuit movement (labeled 1-16). Frame numbers did not appear in the clips. 
Design

The materials were presented in a factorial design with three within-subjects variables: target location (cued or uncued), gaze type (saccade or pursuit), and SOA (125 ms, $333 \mathrm{~ms}$, or $667 \mathrm{~ms}$ ). The dependent variable was the time between the onset of the letter target and the execution of the correct keypress response made in identifying the target.

\section{Procedure}

Each trial began with the presentation of a fixation cross for $1000 \mathrm{~ms}$. Participants were asked to fixate on this cross and to attempt to maintain fixation in the center of the screen throughout each trial. The fixation cross was followed by the presentation of the gaze clip, each of which began with a $1000 \mathrm{~ms}$ display of the gazer staring straight at the participant. This was followed by either a saccadic shift to the left or right, which lasted 125 $\mathrm{ms}$, or a pursuit movement, the duration of which was $667 \mathrm{~ms}$. The target was presented either $125 \mathrm{~ms}, 333 \mathrm{~ms}$ or $667 \mathrm{~ms}$ after the onset of the gaze ${ }^{1}$. Participants were asked to press the appropriate button on the keyboard corresponding to the letter target that was presented. They were asked to respond as quickly and as accurately as possible.

The session began with 16 practice trials, comprising a selection of all possible trial types. This was followed by three further blocks of 64 trials. Thus, there were a total of 198 experimental trials, with 16 trials in each of the 12 cells of the experimental design. Trials were selected randomly within each block. The duration of the entire procedure was approximately 20 minutes.

\section{Results}

Trials involving incorrect responses and those where RTs exceeded 1500 ms or were less than $150 \mathrm{~ms}$, were removed prior to data analysis. This procedure resulted in the 
exclusion of $0.74 \%$ of the data. Mean RTs were computed in each condition of the experiment and the inter-participant means are shown in Figure 2.

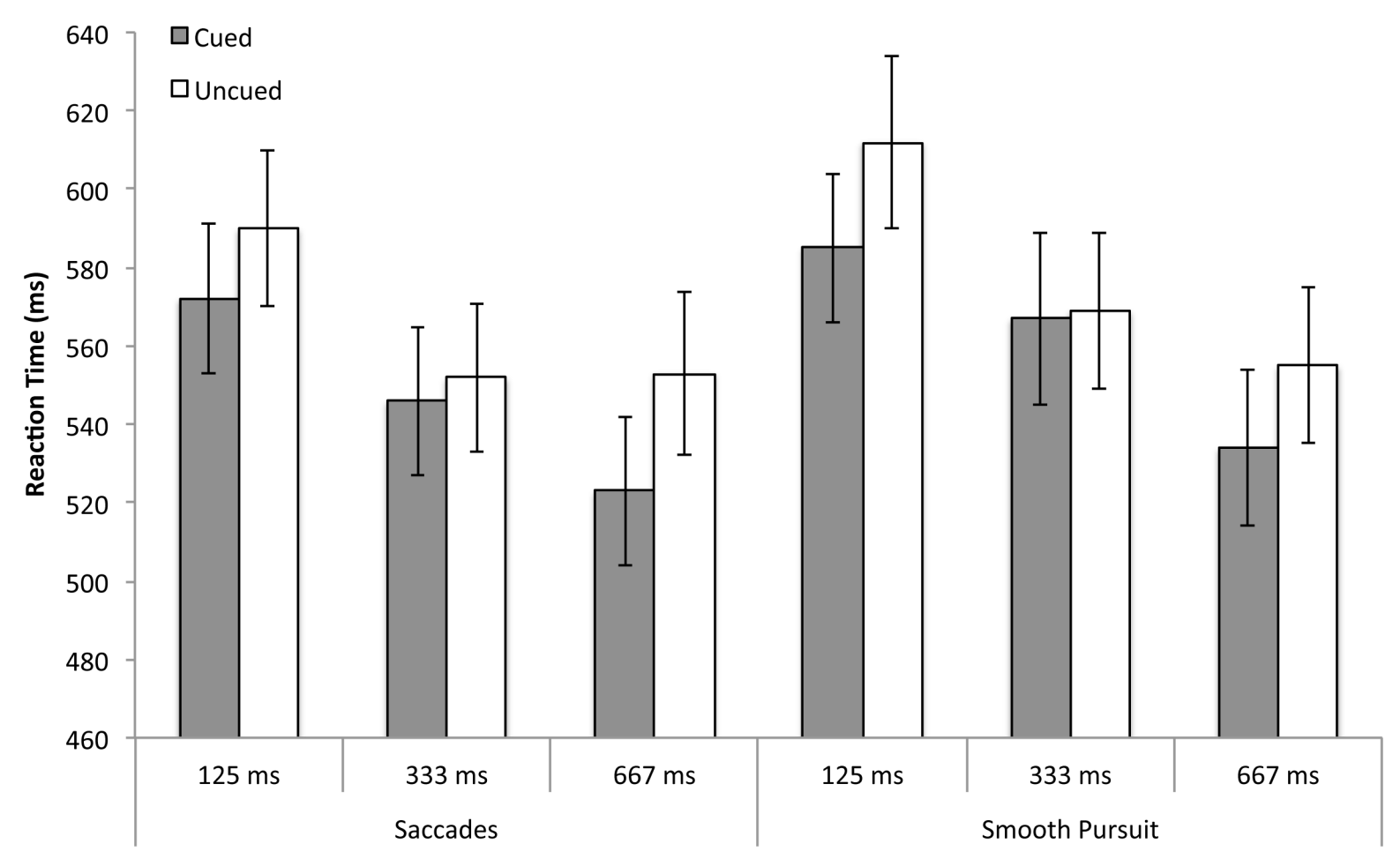

Figure 2. Mean RTs in each condition of Experiment 1. Error bars represent the standard error of the mean.

As can be seen in Figure 2, responses to cued targets were faster than those to uncued targets across all conditions of the experiment. Moreover, the pattern of results for trials involving saccades and pursuit gazes was very similar. An ANOVA conducted on the mean correct RTs with gaze type (saccade vs. pursuit), target location (cued vs. uncued) and SOA (125 ms vs. $333 \mathrm{~ms}$ vs. $667 \mathrm{~ms}$ ) as repeated measures factors supported these observations. This analysis yielded a main effect of gaze type, $F(1,25)=10.40, p<0.01, \eta_{p}{ }^{2}=0.29$, with faster responses on trials involving saccades $(M=556 \mathrm{~ms}, S D=93 \mathrm{~ms})$ versus those involving pursuit gazes $(M=570 \mathrm{~ms}, S D=96 \mathrm{~ms})$; a main effect of SOA, $F(2,50)=39.36$, $p<0.001, \eta_{p}{ }^{2}=0.61$, with RTs decreasing with increasing SOA; and a main effect of target location, $F(1,25)=11.57, p<0.01, \eta_{p}^{2}=0.32$, with faster responses to cued targets $(M=$ 
$555 \mathrm{~ms}, S D=91 \mathrm{~ms})$ than uncued targets $(M=572 \mathrm{~ms}, S D=97 \mathrm{~ms})$. None of the interaction effects reached significance $(p s>0.19)$

Participants made errors on just $3.1 \%$ of trials and there was little evidence of a tradeoff between speed and accuracy (See Table 1). No further analyses were conducted on these data.

Table 1.

Percentage of errors in each condition of Experiment 1

\begin{tabular}{lcccccc}
\hline & \multicolumn{2}{c}{ Saccades } & & \multicolumn{2}{c}{ Pursuit } \\
\cline { 2 - 3 } \cline { 5 - 6 } SOA & Cued & Uncued & & Cued & Uncued \\
\hline $125 \mathrm{~ms}$ & 4.42 & 2.54 & & 3.50 & 1.38 \\
$333 \mathrm{~ms}$ & 3.92 & 4.62 & & 1.85 & 3.27 \\
$667 \mathrm{~ms}$ & 2.35 & 4.27 & & 2.08 & 2.81 \\
\hline
\end{tabular}

\section{Discussion}

Experiment 1 compared gaze-cued orienting in response to saccadic and smooth pursuit gazes and we expected to observe a larger cueing effect for the former than the latter. However, there was no evidence whatsoever for such a difference in this experiment: cueing effects were equivalent for both types of gazes across all the SOAs, suggesting that the attention system is insensitive to the form of a seen eye movement; attention shifts are as likely to be triggered in response to saccadic eye movements as to pursuit gazes.

One striking aspect of the data is that the cueing effect emerges for both types of gazes within $125 \mathrm{~ms}$ of the onset of the gaze cues. While it is well established that gaze-cued orienting can emerge with cue-to-target intervals of as little as 100 ms (Friesen \& Kingstone, 1998; Hill et al., 2010; Langton \& Bruce, 1999), the significance here is that $125 \mathrm{~ms}$ after the onset of each of the gaze cues saccades contain a great deal more information than pursuit gazes (compare the gazes labeled as frame 3 in the top and bottom panels of Figure 1). One might then argue that, while both types of gazes trigger cueing effects of equivalent 
magnitude, pursuit gazes are actually more potent than saccades in that they can initiate attention shifts on the basis of weaker information. Nevertheless, it is clear that by the time that information in the different types of gazes is matched (at the $667 \mathrm{~ms}$ SOA) the cueing effects generated by the two types of gazes remain equivalent; pursuit gazes do not seem to result in the accumulation of greater attentional resources in the cued direction over time than do saccadic gazes, so in this respect they are no more potent than saccadic gaze cues in shifting attention. Instead, it appears to be the case that the attention system deploys resources in the direction signaled by either type of eye movement as soon as the relevant cue is detected.

Another noteworthy observation relating to the time course of the orienting effects generated by the two types of gazes is that the effects were greatest at the shortest and longest SOAs, where they averaged, $23 \mathrm{~ms}$ and $26 \mathrm{~ms}$, respectively, but virtually absent at the intermediate SOA where the magnitude of the effect was just $4 \mathrm{~ms}$. Although the statistical interaction that would license discussion of separate cueing effect at each SOA did not reach significance, the pattern is identical for both types of cue, and so merits some consideration. One possible explanation is that cueing effects at the shortest and longest SOAs reflect the operation of two different forms of orienting. The first is the result of a dedicated system that works to deploy attentional resources automatically in response to seen gazes, while the second is the result of top-down attentional control (i.e., endogenous orienting). That gaze-cued orienting is sub-served by dedicated neural circuitry is suggested by several lines of evidence. For example, Akiyama and colleagues (Akiyama et al., 2006) describe a patient who, following a lesion to the right superior temporal sulcus (STS) - a region of the brain known to be involved in gaze processing (Hoffman \& Haxby, 2000) showed no evidence of gaze-cued orienting, but normal orienting of attention triggered by arrow stimuli. Further support for the idea that gaze-cued orienting is somehow special 
comes from Kingstone et al's report of a split-brain patient who showed the usual gaze cueing effect but only when the gazes were presented to the hemisphere dominant for face processing. Arrows, on the other hand, triggered attention shifts when presented to either hemisphere (Kingstone, Friesen, \& Gazzaniga, 2000). Finally, adaptation to a specific gaze direction has been shown to disrupt gaze-cueing, while adaptation to a pointing stimulus leaves gaze-cueing unchallenged (Bayliss, Bartlett, \& Naughtin, 2011).

The suggestion is therefore that the cueing effect observed at the $125 \mathrm{~ms} \mathrm{SOA}$ in Experiment 1 represents the operation of a fast-acting, automatic mechanism triggered directly by some physical cue or set of cues in the eye-region that correlate with gaze direction (e.g., luminance contrast or the spatial configuration of eye features). Gaze-cued orienting at the longest SOA might be based on the operation of the endogenous system that produces shifts of attention based on current or standing goals (e.g., "look where others look"). Orienting of this form plausibly takes longer to emerge as it requires the processing of the "meaning" of the gaze cues (e.g., encoding a representation with the content "he is looking at that", or "he is looking to the left"). On this account, the magnitude of the cueing effect at the intermediate $333 \mathrm{~ms} \mathrm{SOA}$ is much smaller because by then the automatic effect may have dissipated, while the endogenous effect has yet to emerge.

The main conclusion from Experiment 1 is that gaze-cued orienting is blind to the type of gaze that initiated it. This conclusion is, however, based on the failure to find any higher order interactions involving gaze type and target location in this study. It remains possible that the experiment was insufficiently sensitive to reveal any differences in gazecued orienting between the two types of gazes. It may be, for example, that the reflex-like orienting component, discussed above, is blind to the type of gaze, while the willful component is sensitive to the nature of the eye movement, or vice versa. If both types of attention orienting have similar effects on target detection and operate with time courses that 
overlap more than we have suggested, then Experiment 1 may not tease apart differences between the two types of gazes. Experiment 2 was therefore designed to isolate the stimulusdriven component of gaze-cued orienting and to test whether or not it is sensitive to the type of eye movement.

\section{Experiment 2}

In Experiment 2 participants were told, correctly, that the target to which they were asked to respond was more likely to appear on the opposite side of the screen to that indicated by the direction of gaze. Participants' target detection RTs were measured for targets that appeared in the likely location (i.e., opposite to the direction of gaze), at the gazed-cued location, and at an uncued location (above or below the face), which was not gazed-at, and only as likely to contain a target as the gazed-at location. This method was introduced by Friesen and colleagues (Friesen et al., 2004) and it allowed us to test whether attention is simultaneously drawn to the gazed-at location (e.g., by reflexive gaze-cued orienting) and the likely location (e.g., by endogenous orienting) for both saccadic gazes and pursuit gazes. As in Experiment 1, we also explored the time-course of the effect; however, in order to keep the duration of the procedure similar to that of Experiment 1, we used only $125 \mathrm{~ms}$ and $667 \mathrm{~ms}$ SOAs, which yielded gaze cueing effects of the largest magnitude in Experiment 1. The type of gaze was manipulated as a between-subjects variable in Experiment 2, again to minimize the duration of the procedure.

If saccadic gazes are a more potent cue for reflexive orienting, we might expect to observe gaze-cued orienting effects for these gazes, with a much weaker or absent effect for pursuit gazes. Endogenous orienting effects in the opposite direction to the gaze cues were expected in response to both gaze types, and were predicted to be greater in magnitude at the longer SOA as willful orienting typically has a slower rise time than its stimulus-driven counterpart (Müller \& Rabbitt, 1989). Finally, because participants might be expected to 
maintain a goal specifying that attention should be oriented in the opposite direction to the perceived gaze, endogenous shifts of attention in the actual direction of the seen gaze were not expected to occur. In other words, the gaze cueing effect observed at the longer SOA in Experiment 1 was not predicted in Experiment 2.

\section{Method}

\section{Participants}

The participants in Experiment 2 were 48 undergraduates at the University of Stirling, from all of whom written informed consent was obtained. Participants were randomly allocated to either the saccadic or the pursuit gaze conditions, under the constraint that an equal number were allocated to each group. The participants viewing the saccadic gazes had an average age of 22.8 years (range 17-56) and the group comprised 18 females and 6 males. Participants allocated to the pursuit gaze condition had an average age of 20 years (range 17-37) and the group consisted of 20 females and 4 males.

\section{Materials and apparatus}

The video clips recorded for use in Experiment 1 were also used in Experiment 2. The target stimulus consisted of an asterisk in 18 point Arial font, which could appear either $6^{\circ}$ to the left or right of the centre of the screen for gaze-cued and expected trials, or $6^{\circ}$ vertically above or below fixation in the case of trials in the uncued conditions. The target stimulus was white against a black background. Only one type of target was used in this experiment as the task was changed from target discrimination (T versus F) in Experiment 1, to target detection in Experiment 2. Because targets could now appear above and below the face as well as on its left and right, the use of a $2 \mathrm{AFC}$ discrimination task with response keys aligned horizontally would introduce a confound where responses to targets aligned in

one direction (e.g., the horizontal) would be subject to the Simon effect (Simon \& Rudell, 1967), whereas those in the other would not. This would be particularly problematic in this 
experiment because targets aligned vertically represent uncued locations, whereas those aligned horizontally represent the expected and gaze-cued locations. The use of a simple keypress response upon detection of a single target stimulus eliminates this potential confound.

Stimuli were displayed on a Lenovo 19 inch monitor and their presentation was controlled using E-Prime software. Responses were collected using a Serial Response Box (Psychology Software Tools, inc.).

\section{Design}

In Experiment 2, target location (gaze-cued, expected, or uncued) and SOA (125 ms or $667 \mathrm{~ms}$ ) were within-subjects variables and gaze type (saccadic or pursuit gaze) was a between-subjects variable. Reaction time was recorded as the dependent variable.

\section{Procedure}

Trials began with a fixation cross presented on the screen for $1000 \mathrm{~ms}$. As in Experiment 1, participants were asked to maintain fixation on this cross for the duration of each trial. The cross was followed by the appearance of a face, gazing straight ahead for a further $1000 \mathrm{~ms}$. Next, the face either made a saccadic gaze shift lasting $125 \mathrm{~ms}$ or a pursuit movement lasting $667 \mathrm{~ms}$. The target then appeared after a delay determined by the SOA condition (either $125 \mathrm{~ms}$ or $667 \mathrm{~ms}$ after the onset of either type of gaze cue). The target could appear in one of the following four locations: the location toward which the gaze was directed (the gaze-cued location); the location opposite the gazed-at location (the expected location); above, or below the face (uncued locations). On catch trials, no target was presented. Participants were asked to respond by pressing the central button on the response box as soon as they detected the target, and to withhold a response if no target appeared. They were also informed that the target was four times more likely to appear in the opposite location to the one indicated by the gaze. 
Under each of the SOA conditions there were 16 trials where the target appeared in a gaze-cued location, 64 trials where the target appeared in the expected location, and a total of 32 trials where the target was uncued (16 trials where the target appeared above the face, and 16 where it appeared below the face), giving a total of 224 target present trials. There were also 32 catch trials in the experiment in which no target was presented. The grand total of 256 trials was divided into 4 blocks of 64 trials in each of which trials were randomly selected, without replacement, from the total pool of 256 trials. Participants also completed a block of 16 practice trials before the experimental trials. As in Experiment 1, the entire procedure lasted around 20 minutes.

\section{Results}

Participants incorrectly responded to just $1.95 \%$ of catch trials and failed to respond on just $0.2 \%$ of trials where the target was present. Individual trials where responses were faster than $150 \mathrm{~ms}$ or longer than $1000 \mathrm{~ms}$ were discarded. This resulted in the loss of $0.70 \%$ of the data ${ }^{2}$. The mean RTs for each participant in each condition of the experiment were then computed and these data are summarized in Figure 3.

As can be seen in Figure 3, on average participants' responses were faster for gazecued targets than for uncued targets for both types of gazes and at each of the SOAs. The detection of targets at expected targets was also faster than those at uncued locations for both types of gazes, with a rather larger difference apparent at the longer SOA. It is also clear from the figure that the pattern of data for saccades and pursuit gazes is virtually identical. 


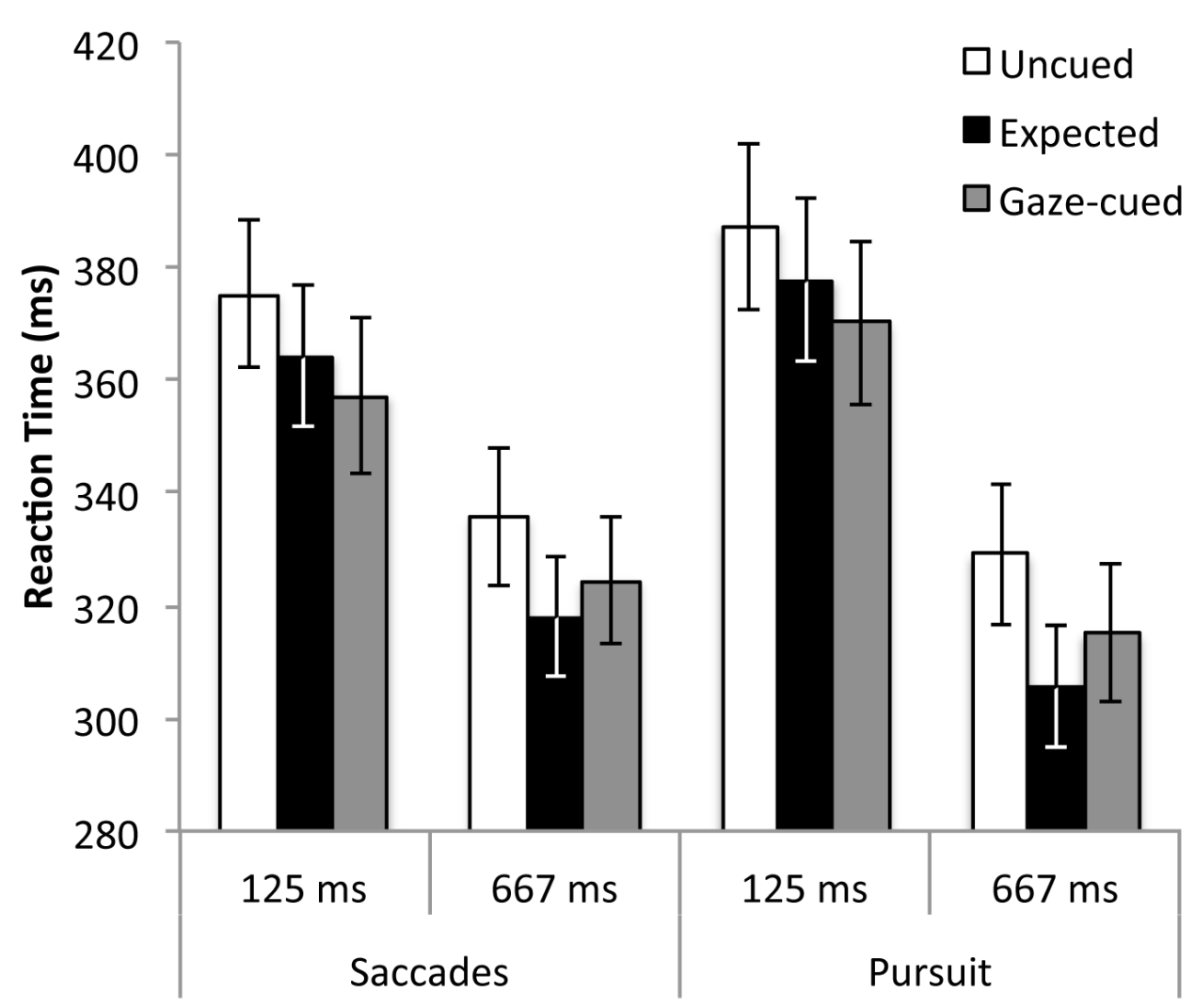

Figure 3. Mean RTs in each condition of Experiment 2. Error bars represent the standard error of the mean.

The RT data were entered into an ANOVA with target location and SOA as withinsubjects factors and gaze type as a between-subjects factor. In support of the above observations, this analysis yielded main effects of target location, $F(2,92)=23.75, p<.01$, $\eta_{p}{ }^{2}=0.34$, and SOA, $F(1,46)=162.39, p<.01, \eta_{p}{ }^{2}=0.78$, and a significant interaction between these factors, $F(2,92)=7.59, p<.01, \eta_{p}{ }^{2}=0.14$. The relevant data for this interaction are summarized in Figure 4. Its source appears to be larger gaze cueing (i.e., the difference between RTs to uncued and gaze-cued targets) than expectancy cueing (i.e., the difference between RTs to uncued and expected targets) at the short SOA, but the opposite pattern at the longer SOA. Follow-up analyses of this interaction were conducted by testing for simple main effects of target location on RT at each of the SOA conditions. 
At the short, $125 \mathrm{~ms}$ SOA, the simple main effect of target location was significant, $F(2,184)=14.68, p<.01$. Fisher's LSD tests confirmed that expected targets $(M=371 \mathrm{~ms}$, $S D=67 \mathrm{~ms})$ were responded to faster than uncued targets $(M=381 \mathrm{~ms}, S D=67 \mathrm{~ms}), t(184)$ $=3.13, p<.01$, as were gaze-cued targets $(M=364 \mathrm{~ms}, S D=69 \mathrm{~ms}), t(184)=5.40, p<.01$. At this SOA, the cueing effects were therefore both significant but larger for gaze-cued targets $(17 \mathrm{~ms})$ than for expected targets $(10 \mathrm{~ms})$.

The simple main effect of target location was also significant at the $667 \mathrm{~ms} \mathrm{SOA}$, $F(2,184)=20.59, p<.01$. Once again, expected targets $(M=312 \mathrm{~ms}, S D=52 \mathrm{~ms})$ received faster responses than uncued targets $(M=332 \mathrm{~ms}, S D=60 \mathrm{~ms}), t(184)=6.36, p<.01$, as did gaze-cued targets $(M=320 \mathrm{~ms}, S D=57 \mathrm{~ms}), t(184)=3.94, p<.01$. Again, the cueing effects were significant at this SOA, but here they were larger for expected targets $(20 \mathrm{~ms})$ than gaze-cued targets $(12 \mathrm{~ms})$, which is the opposite pattern of cueing effects to those observed at the shorter SOA.

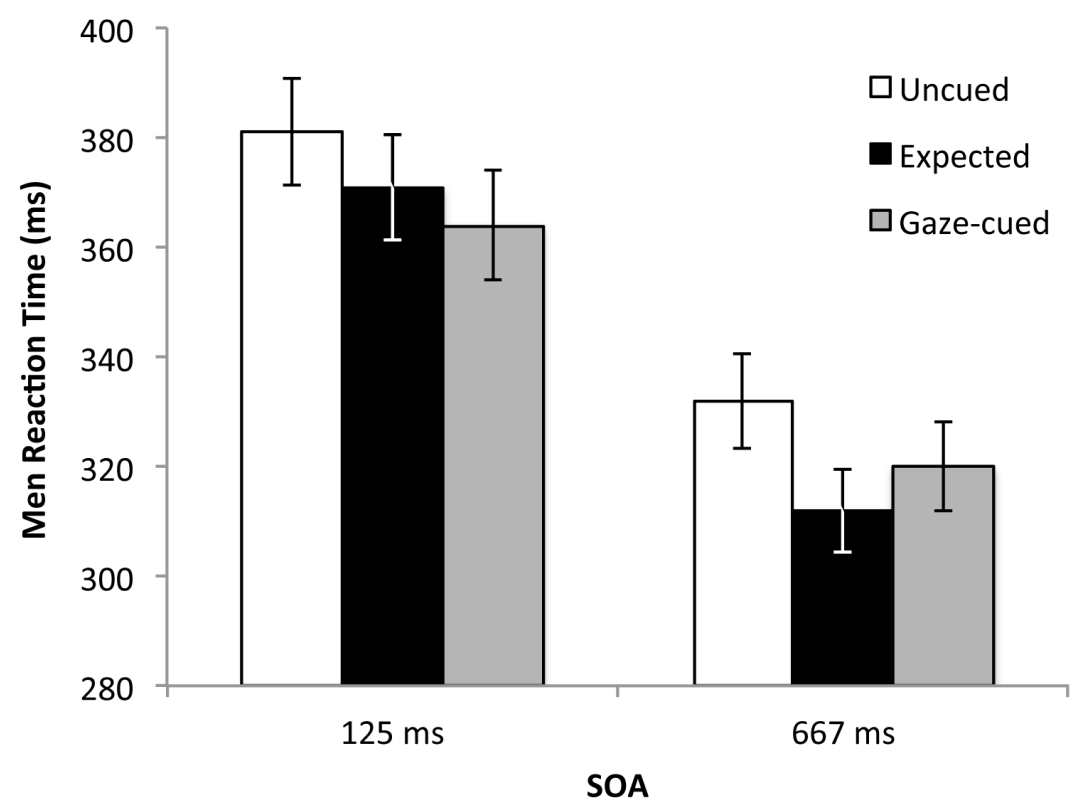

Figure 4. Mean target detection RTs as a function of target location and SOA in Experiment 2. Error bars represent the standard error of the mean. 
The overall ANOVA also yielded a significant interaction between SOA and gaze type, $F(1,46)=7.78, p<.01, \eta_{p}^{2}=0.15$. This interaction appears to have arisen because the usual forewarning effect (faster RTs with increasing SOAs) was larger for participants viewing pursuit gazes than for those viewing saccadic gazes. No other main effects or interactions reached significance $(p \mathrm{~s}>.68)$

\section{Discussion}

The pattern of results in Experiment 2 is clear: both types of gazes resulted in equivalent gaze-cued orienting effects (faster RTs at cued versus uncued locations) across each of the SOA conditions. Participants were also able to deploy attention willfully and simultaneously in the opposite direction to the seen gazes, and they were able to do this as readily in response to a saccadic gaze as to a pursuit gaze. The gaze cueing effect was observed as rapidly as $125 \mathrm{~ms}$ after gaze onset, and was reduced, but still present, by $667 \mathrm{~ms}$. In contrast, the willful, endogenous cueing effect was smaller (but statistically significant) at the shortest SOA, and increased in magnitude at the $667 \mathrm{~ms} \mathrm{SOA}$. The observation of equivalent gaze cueing effects for saccadic and pursuit gazes was again contrary to predictions, and supports the conclusion of Experiment 1 that gaze-cued orienting is insensitive to the speed of eye movements.

The time-course of the gaze cueing effect also supports the suggestion that the effect observed at the shortest SOA in Experiment 1 is the result of a fast-rising automatic orienting mechanism that serves to deploy attention in the direction of perceived gaze, again regardless of the nature of the eye movement. However, contrary to the experimental prediction, the gaze-cueing effect persisted for as long as $667 \mathrm{~ms}$ after the onset of the gaze cue. To the extent that Experiment 2 isolates a purely stimulus-driven form of attention (i.e., because participants demonstrably orient attention endogenously in the opposite direction to the seen gaze), this observation runs counter to the suggestion made in the discussion of 
Experiment 1 that this form of gaze-cued orienting is fast-rising but short-lived. We return to this issue in the General Discussion.

The fact that attention can be deployed voluntarily in the opposite direction to eye movements, and simultaneously in the same direction as indicated by a gaze supports the findings of (Friesen et al., 2004) who showed a similar pattern of data using the same method (Hayward \& Ristic, 2013; see also Tipples, 2008). The point we are making here, however, is that having isolated the reflex-like component of gaze-cued attention by making gazes counterpredictive of the target location, both saccades and pursuit gazes were equally potent in initiating attention shifts. Again, the conclusion is that the nature of the gaze shift is irrelevant to the attention system.

\section{General Discussion}

The experiments reported in this paper were designed to test whether the kind of movement made by the eyes impacts upon gaze-cued orienting of attention. Contrary to predictions, participants were insensitive to how the eyes moved, at least with regard to saccades and smooth pursuit movements of the eyes. These gazes produced cueing effects of equal magnitude in Experiment 1, and each type of gaze was capable of triggering an attention shift in the gazed-at direction while at the same time participants' willful attention was directed towards a different location (Experiment 2). The latter result strongly suggests that the automatic component of gaze-cued orienting is blind to the nature of eye movements.

While the findings reported here suggest that the type of eye movement has no impact on gaze-cued orienting, they do not allow us to conclude that gaze cued attention is insensitive to motion per se. For it may be that the cueing effects generated in Experiments 1 and 2 were the result of attention shifts in the direction of the perceived gaze movement, whatever the type, rather than the result of any computation of a precise line of regard from 
static cues. The fact that in both experiments, pursuit gazes were able to trigger cueing effects as early as $125 \mathrm{~ms}$ following the onset of the cue makes a reasonable case for this. At this point in time, the iris/pupil region is only very slightly displaced from straight ahead (see frame 3 in the bottom panel of Figure 1), and when presented in isolation as a static stimulus, participants are unable to decide whether it represents a direct or averted gaze; however, people have no difficulty judging the motion direction of the eyes when shown two successive frames from the pursuit gaze $\operatorname{clips}^{3}$. So, at $125 \mathrm{~ms}$ after the onset of a pursuit gaze, the static configuration of the eye features, or the luminance distribution within the eye may be insufficient to signal that the gaze is deviated to the left of straight ahead. However, a change in location of the iris to the left by an even smaller degree - perhaps signaled by a change in either or both of the luminance or configural information - provides an unambiguous cue to the side of space in which the gaze is directed. It is therefore clearly possible that the attention system uses the latter information to issue in a covert deployment of attention. Whether this is actually the case is an empirical issue. The findings of Experiments 1 and 2 do, however, suggest that the rate of displacement of the relevant cues has little effect on gaze-cued attention.

We have argued that the effects observed in Experiments 1 and 2 are best explained by the operation of a fast acting stimulus-driven mechanism that orients attention automatically in the direction of gaze based on the detection of some feature, or set of features, in the eye region that correlate with the direction of gaze. These could be static cues to gaze direction, or cues from the motion of iris/pupil region. This can explain the observation of gaze-cued attention at the $125 \mathrm{~ms} \mathrm{SOA}$ in both experiments. Moreover, the reliable gaze-cued orienting effect at the $667 \mathrm{~ms}$ SOA in Experiment 2 plausibly suggests that this effect is quite long-lived; in this experiment endogenous attention was presumably occupied at the opposite location to that indicated by the gaze, which participants knew was 
likely to contain a target, so that any gaze cueing effects were likely generated by a stimulusdriven, as opposed to a goal-driven mechanism.

However, the conclusion that the stimulus-driven gaze-cueing effect is fast-rising and persists for as long as $667 \mathrm{~ms}$ runs into difficulty when faced with the observation that the magnitude of the cueing effect at the $333 \mathrm{~ms}$ SOA of Experiment 1 was very small indeed. One way to reconcile these observations is to suggest that the cueing effects observed at 667 ms are not the results of a long-lived, purely stimulus-driven mechanism, but are instead generated by what has been termed automated symbolic orienting (Ristic \& Kingstone, 2012). Ristic and Kingstone (2012) argue that this form of attentional orienting is independent of exogenous and endogenous orienting and is engaged by stimuli such as gazes, arrows and words with spatial meaning; it occurs without intention, and arises because of the overlearning of associations between cues and target events. One explanation for our data is therefore that the fast-rising effect is based on a low-level reflex, which is indeed short lived, decaying by $333 \mathrm{~ms}$, while the effect at $667 \mathrm{~ms}$ is based on automatic symbolic orienting, which takes longer to emerge, but persists for at least $667 \mathrm{~ms}$. It may even be that the fast acting effect exerts some inhibition on automated symbolic orienting, which accounts for the slower rise time of the effect here than has been observed in work with arrows as symbolic cues (Ristic \& Kingstone, 2012; Ristic, Landry, \& Kingstone, 2012). It should be noted, however, that the suggestion of a two systems account ultimately rests on a non-significant interaction between SOA and target type in Experiment 1. A more parsimonious explanation is that the effects are based on a unitary, fast, and relatively longlived form of orienting. Whether this is some general type of automated symbolic orienting, a gaze-specific mechanism triggered directly by eye-features (e.g., their configuration or movement direction), or indeed one induced by motion direction per se clearly requires further research. 
Although participants in Experiments 1 and 2 were asked to maintain fixation in the centre of the screen for the duration of each trial, this was not actually monitored. It is therefore possible that the data are somewhat contaminated by trials where participants may have made saccadic eye movements in response to the cueing stimuli. It is unlikely that this is a problem for the interpretation of the cueing effects obtained for the $125 \mathrm{~ms}$ SOAs, as this is too short a duration to program and execute any kind of eye movement; however, a cue-totarget duration of $667 \mathrm{~ms}$ would be sufficient for cue-driven saccades to have an impact. Making a saccade in the direction of a gaze in Experiment 1 would bring the target on a cued trial closer to the fovea, potentially contributing to the RT advantage for cued over uncued trials. In Experiment 2 it may even be beneficial for participants to strategically saccade in the opposite direction to perceived gaze movements in anticipation of targets that are likely to appear in these locations. The effect of this would be to enhance the endogenous cueing effect as a target in the expected location would be brought closer to the fovea than it would otherwise be were fixation to be maintained centrally.

However, there are reasons to believe that these kinds of cue-driven eye movements play only a small part, if any, in gaze-cued orienting of attention. For example, Mansfield, Farroni, and Johnson (2003) eye-tracked participants in a gaze-cueing study and showed that although participants did tend to make a number of saccades in response to gaze cues, removal of these trials from the data did not influence the magnitude of the cueing effect. It therefore seems unlikely that eye movements make a telling contribution to the effects observed in Experiment 1. It is possible, however, that strategic saccades made in the opposite direction to the gaze cues in Experiment 2 may explain the larger magnitude of the endogenous cueing effect observed at the $667 \mathrm{~ms}$ SOA than at the $125 \mathrm{~ms}$ SOA of this experiment. For it is easily possible to program and execute a strategic saccade toward a likely target location within $667 \mathrm{~ms}$ of cue onset, but not within $125 \mathrm{~ms}$. Targets appearing 
in the expected location at the longer SOA may therefore receive an extra benefit from being foveated on some proportion of trials as well as being the recipients of endogenous attention; targets appearing after the shorter cue-to-target interval, on the other hand, would only benefit from being endogenously attended.

However, it is notable that even if participants did adopt the strategy of making saccades towards likely target locations in Experiment 2, the data clearly suggest that they nevertheless also allocated covert attention toward the gazed-at location. Indeed, it may be that the experiment underestimates the magnitude of gaze-cued orienting. For on trials where participants do make strategic saccades, targets that subsequently appear in gaze-cued locations will actually be positioned further from the participants' fovea than on trials where no such strategic saccades are made. In addition, there is no reason to suggest that participants might be more likely to adopt this strategy for one type of seen gaze rather than another, so it is unlikely that our main conclusion should be compromised by a lack of control over this factor.

A second potential limitation in our studies is the fact that the identity of the gazer was likely to have been known by some of the participants. This is because it is know that, for female participants, gaze cueing is enhanced for personally familiar versus unfamiliar faces (Deaner, Shepherd, \& Platt, 2007). As the samples in both experiments contained a majority of female participants, it is at least possible that the results over-estimate the magnitude of gaze-cued orienting in the general population. We did not collect the relevant data from our participants, so we cannot categorically rule out this possibility, but again there is no reason to believe that familiarity with the gazer will differentially influence attentional orienting to saccadic and pursuit gazes. So while the overall magnitude of gaze cueing might be overestimated, the fact that some of our participants may have known the 
gazer does not force us to re-evaluate the conclusion that gaze-cued orienting is insensitive to the type of eye motion that triggered the effect.

In each of the experiments reliable gaze cueing effects were obtained for both types of gazes even though the tasks in these studies were rather different: Experiment 1 required participants to make a two choice target discrimination response, whereas Experiment 2 demanded a simple target detection response. This might be important because it is known that the time course of exogenous orienting of attention triggered by peripheral onsets is sensitive to task demands (Lupiáñez, Milán, Tornay, \& Madrid, 1997). Had we used target detection instead of discrimination in Experiment 1, or retained the target discrimination task in Experiment 2 perhaps we would have observed differences in cueing effects generated by saccades and pursuit gazes (e.g., earlier onset of the former relative to the latter). This seems unlikely, however, because there is no evidence to suggest that gaze-cued orienting is sensitive to the same kinds of task demands as orienting toward peripheral onsets: gaze cued orienting is reliably generated at SOAs of as little as $100 \mathrm{~ms}$ and persists for at least $700 \mathrm{~ms}$ whether the task involves target detection or target discrimination (Driver et al., 1999; Friesen \& Kingstone, 1998). It is of course possible that a systematic manipulation of task type and SOA would reveal the higher order interaction that would support this suggestion, but we are more persuaded by the striking similarity in the patterns of gaze-cueing effects across two experiments involving different tasks: reliable gaze-cued orienting effects at SOAs as short as $125 \mathrm{~ms}$ and as long as $667 \mathrm{~ms}$.

In summary, the studies presented here suggest the manner in which the eyes move does not contribute to gaze-cued orienting of attention, although it seems likely that motion direction may be sufficient to trigger the effect. Any flexibility in the system that would, for example, prevent a viewer from shifting attention in response to another person's gaze must come from sensitivity to other physical cues, such as the timing of fixations, or contextual 
information such as head movements accompanying gaze shifts or the concurrent verbal context. These suggestions await further investigation, which may benefit from the use of more realistic stimuli and methods that allow them to be used in controlled, but more naturalistic contexts (Risko, Richardson, \& Kingstone, 2016; Schilbach et al., 2013). 


\section{Footnotes}

1. With dynamic stimuli that unfold at different speeds, if SOA is to be independently manipulated, there is an inevitable confound between gaze-type and inter-stimulus interval (ISI) - the time between the endpoint of the gaze and the onset of the target. For example, in trials where the SOA is $667 \mathrm{~ms}$, the ISI for saccadic gazes is therefore $542 \mathrm{~ms}$ and $0 \mathrm{~ms}$ for the smooth pursuit gazes. However, a pilot study $(\mathrm{N}=16)$ that tested for gaze cued orienting from the different gazes with a fixed ISI of $667 \mathrm{~ms}$ yielded an identical pattern of results to those obtained here.

2. The upper bound on the cut-off for treating RTs as outliers in Experiment 2 (1000 ms) was faster than in Experiment $1(1500 \mathrm{~ms})$ because the simple detection task used in Experiment 2 typically attracts faster RTs than the target identification task used in Experiment 1. The percentages of data removed in each case were similar $(0.74 \%$ in Experiment 1, 0.70\% in Experiment 2). In any case, the pattern of data in each of Experiments 1 and 2 are identical, although somewhat nosier, if the RT data are untrimmed and median RTs are used to summarise each participant's performance.

3. In support of this observation, of a sample of 10 participants shown frame 3 from figure 1 (the displacement of the eyes $125 \mathrm{~ms}$ after the onset of the gaze), five judged the gaze direction to be straight ahead, and five judged it to be averted. Each of a different sample of 10 participants shown frame 2 for $1000 \mathrm{~ms}$ immediately followed by frame 3 judged that the gaze had shifted to their left as opposed to shifting to their right or remaining unchanged. Gaze direction is clearly evident from the motion signal produced by presenting adjacent frames in this way so as to simulate motion; however, without this motion signal, the displacement of the eyes from straight ahead is difficult to perceive. 


\section{References}

Akiyama, T., Kato, M., Muramatsu, T., Saito, F., Umeda, S., \& Kashima, H. (2006). Gaze but not arrows: A dissociative impairment after right superior temporal gyrus damage. Neuropsychologia, $\quad 44(10), \quad 1804-1810$. http://doi.org/10.1016/j.neuropsychologia.2006.03.007

Ando, S. (2004). Perception of gaze direction based on luminance ratio. Perception, 33, 1173-1184. http://doi.org/10.1068/p5297

Anstis, S. M., Mayhew, J. W., \& Morley, T. (1969). The perception of where a face or television "portrait" is looking. The American Journal of Psychology, 82(4), 474-489.

Argyle, M. (1988). Bodily Communication (2nd ed.). London: Methuen \& Co. Ltd.

Bayliss, A. P., \& Tipper, S. P. (2006). Predictive gaze cues and personality judgments should eye trust you? Psychological Science, 17, 514-520. http://pss.sagepub.com/content/17/6/514.short

Bayliss, A. P., Bartlett, J., \& Naughtin, C. K. (2011). A direct link between gaze perception and social attention. Journal of Experimental Psychology: Human Perception and Performance, 37(3), 634-644. http://doi.org/10.1037/a0020559

Bayliss, A. P., Griffiths, D., \& Tipper, S. P. (2009). Predictive gaze cues affect face evaluations: The effect of facial emotion. European Journal of Cognitive Psychology, 21(7), 1072-1084. http://doi.org/10.1080/09541440802553490

Bobak, A. K., \& Langton, S. R. H. (2015). Working memory load disrupts gaze-cued orienting of attention. Frontiers in Psychology, 6:1258. http://doi.org/10.3389/fpsyg.2015.01258

Calder, A. J., Beaver, J. D., Winston, J. S., \& Dolan, R. J. (2007). Separate coding of different gaze directions in the superior temporal sulcus and inferior parietal lobule. 
Current Biology, 17, 20-25. http://doi.org/10.1016/j.cub.2006.10.052

Cline, M. G. (1967). The perception of where a person is looking. The American Journal of Psychology, 80(1), 41-50. http://www.jstor.org/stable/1420539

Deaner, R. O., Shepherd, S. V., \& Platt, M. L. (2007). Familiarity accentuates gaze cuing in women but not men. Biology Letters, 3(1), 65-68. http://doi.org/10.1098/rsbl.2006.0564

Driver, J., Davis, G., Ricciardelli, P., Kidd, P., Maxwell, E., \& Baron-Cohen, S. (1999). Gaze perception triggers reflexive visuospatial orienting. Visual Cognition, 6(5), 509540. http://doi.org/10.1080/135062899394920

Friesen, C. K., \& Kingstone, A. (1998). The eyes have it! Reflexive orienting is triggered by nonpredictive gaze. Psychonomic Bulletin \& Review, 5(3), 490-495. http://link.springer.com/article/10.3758/BF03208827

Friesen, C. K., Ristic, J., \& Kingstone, A. (2004). Attentional effects of counterpredictive gaze and arrow cues. Journal of Experimental Psychology: Human Perception and Performance, 30(2), 319-329. http://doi.org/10.1037/0096-1523.30.2.319

Frischen, A., Bayliss, A. P., \& Tipper, S. P. (2007). Gaze cueing of attention: visual attention, social cognition, and individual differences. Psychological Bulletin, 133(4), 694-724. http://doi.org/10.1037/0033-2909.133.4.694

Gibson, J. J., \& Pick, A. D. (1963). Perception of another person's looking behavior. The American Journal of Psychology, 76(3), 386-394. http://www.jstor.org/stable/1419779

Hayward, D. A., \& Ristic, J. (2013). The uniqueness of social attention revisited: working memory load interferes with endogenous but not social orienting. Experimental Brain Research, 231(4), 405-414. http://doi.org/10.1007/s00221-013-3705-z

Hill, J. L., Patel, S., Gu, X., Seyedali, N. S., \& Bachevalier, J. (2010). Social orienting: 
reflexive versus voluntary control. Vision Research, 50, 2080-2092. http://www.sciencedirect.com/science/article/pii/S0042698910003676

Hoffman, E. A., \& Haxby, J. V. (2000). Distinct representations of eye gaze and identity in the distributed human neural system for face perception. Nature Neuroscience, 3(1), 8084. http://www.nature.com/neuro/journal/v3/n1/abs/nn0100_80.html

Jenkins, R., Beaver, J. D., \& Calder, A. J. (2006). I thought you were looking at me: direction-specific aftereffects in gaze perception. Psychological Science, 17(6), 506513. http://doi.org/10.1111/j.1467-9280.2006.01736.x

Kendon, A. (1967). Some functions of gaze-direction in social interaction. Acta Psychologica, 26 , $22-63$. http://www.sciencedirect.com/science/article/pii/0001691867900054

Kingstone, A., Friesen, C. K., \& Gazzaniga, M. S. (2000). Reflexive joint attention depends on lateralized cortical connections. Psychological Science, 11(2), 159-166. http://pss.sagepub.com/content/11/2/159.short

Lachat, F., Conty, L., Hugueville, L., \& George, N. (2012). Gaze cueing effect in a face-toface situation. Journal of Nonverbal Behavior, 36, 177-190. http://doi.org/10.1007/s 10919-012-0133-x

Langton, S. R. H., \& Bruce, V. (1999). Reflexive visual orienting in response to the social attention of others. Visual Cognition, 6(5), 541-567. http://www.tandfonline.com/doi/abs/10.1080/135062899394939

Langton, S. R. H., McIntyre, A. H., \& Hancock, P. J. B. (2017). Covert and overt orienting of attention in response to naturalistic, dynamic shifts of another's social gaze. Manuscript submitted for publication.

Langton, S. R. H., Watt, R. J., \& Bruce, V. (2000). Do the eyes have it? Cues to the direction 
of social attention. Trends in Cognitive Sciences, 4(2), 50-59. http://www.sciencedirect.com/science/article/pii/S1364661399014369

Law, A. S., Langton, S. R. H., \& Logie, R. H. (2010). Assessing the impact of verbal and visuospatial working memory load on eye-gaze cueing. Visual Cognition, 18(10), 14201438. http://doi.org/10.1080/13506285.2010.496579

Lupiáñez, J., Milán, E. G., Tornay, F. J., \& Madrid, E. (1997). Does IOR occur in discrimination tasks? Yes, it does, but later. Perception \& Psychophysics, 59, 12411254. http://www.springerlink.com/index/E715V114L5523P53.pdf

Mansfield, E., Farroni, T., \& Johnson, M. (2003). Does gaze perception facilitate overt orienting? Visual Cognition, 10(1), 7-14. http://doi.org/10.1080/13506280143000647

Müller, H. J., \& Rabbitt, P. M. (1989). Reflexive and voluntary orienting of visual attention: time course of activation and resistance to interruption. Journal of Experimental Psychology: Human Perception and Performance, 15(2), 315-330. http://psycnet.apa.org/journals/xhp/15/2/315/

Posner, M. I. (1980). Orienting of attention. Quarterly Journal of Experimental Psychology, 32(1), 3-25. http://doi.org/10.1080/00335558008248231

Quadflieg, S., Mason, M. F., \& Macrae, C. N. (2004). The owl and the pussycat: Gaze cues and visuospatial orienting. Psychonomic Bulletin \& Review, 11(5), 826-831. http://link.springer.com/article/10.3758/BF03196708

Risko, E. F., Richardson, D. C., \& Kingstone, A. (2016). Breaking the fourth wall of cognitive science: Real-world social attention and the dual function of gaze. Current Directions $\quad$ in $\quad$ Psychological $\quad$ Science, $\quad 25(1), \quad 70-74$. http://doi.org/10.1177/0963721415617806

Ristic, J., \& Kingstone, A. (2005). Taking control of reflexive social attention. Cognition, 
94, B55-B65. http://doi.org/10.1016/j.cognition.2004.04.005

Ristic, J., \& Kingstone, A. (2012). A new form of human spatial attention: automated symbolic orienting. Visual Cognition, 20(3), 244-264. http://doi.org/10.1080/13506285.2012.658101

Ristic, J., Landry, M., \& Kingstone, A. (2012). Automated symbolic orienting: The missing link. Frontiers in Psychology, 3:560. http://doi.org/10.3389/fpsyg.2012.00560/abstract

Schilbach, L., Timmermans, B., Reddy, V., Costall, A., Bente, G., Schlicht, T., \& Vogeley, K. (2013). Toward a second-person neuroscience. Behavioral and Brain Sciences, 36(4), 393-414. http://doi.org/10.1017/S0140525X12000660

Schuller, A. M., \& Rossion, B. (2001). Spatial attention triggered by eye gaze increases and speeds up early visual activity. Neuroreport, 12(11), 2381-2386. http://journals.lww.com/neuroreport/Abstract/2001/08080/Spatial_attention_triggered_b y_eye_gaze_increases.19.aspx

Schuller, A. M., \& Rossion, B. (2004). Perception of static eye gaze direction facilitates subsequent early visual processing. Clinical Neurophysiology, 115, 1161-1168. http://doi.org/10.1016/j.clinph.2003.12.022

Simon, J. R., \& Rudell, A. P. (1967). Auditory S-R compatibility: The effect of an irrelevant cue on information processing. Journal of Applied Psychology, 51(3), 300-304. http://doi.org/10.1037/h0020586

Tipples, J. (2008). Orienting to counterpredictive gaze and arrow cues. Perception \& Psychophysics, 70(1), 77-87. http://doi.org/10.3758/PP.70.1.77

Watt, R., Craven, B., \& Quinn, S. (2007). A role for eyebrows in regulating the visibility of eye gaze direction. The Quarterly Journal of Experimental Psychology, 60(9), 11691177. http://doi.org/10.1080/17470210701396798 
Wiese, E., Zwickel, J., \& Müller, H. J. (2013). The importance of context information for the spatial specificity of gaze cueing. Attention, Perception, \& Psychophysics, 75(5), 967982. http://doi.org/10.3758/s13414-013-0444-y 